\title{
Pulse Wave Velocity as an Indicator of Atherosclerosis in Obstructive Sleep Apnea Syndrome Patients
}

\author{
Hiroyuki Nagahama, Masatada SoeJima, Hitoshi UenOMACHI*, Youichirou Higashi, \\ Katsuhiko Yotsumoto, Takuya SAMUKAwA and Terukatsu ARIMA
}

\begin{abstract}
Objective Obstructive sleep apnea syndrome (OSAS) is associated with increased cardiovascular morbidity and mortality. We investigated the values of brachialankle pulse wave velocity as an indicator of atherosclerosis in obstructive sleep apnea syndrome patients.

Materials and Methods Brachial-ankle pulse wave velocity (baPWV) was measured in 104 OSAS patients and 104 healthy control subjects matched for age, sex, and body mass index (BMI). BaPWV values were compared in both groups and investigated with respect to the number of risk factors for atherosclerosis, including hypertension, hypercholesterolemia, impaired glucose tolerance, smoking, and obesity. Comparisons were also made between 48 OSAS group cases and 90 control group cases free from hypertension, which has a major impact on baPWV.

Results As compared to the control group, the OSAS group had significantly higher baPWV $(1,645 \pm 349 \mathrm{~cm} / \mathrm{s}$ vs $1,436 \pm 278 \mathrm{~cm} / \mathrm{s}, p<0.0001$ ), and values obtained for baPWV were significantly higher in the OSAS group than in the control group even in groups free from hypertension $(1,453 \pm 216 \mathrm{~cm} / \mathrm{s}$ vs $1,374 \pm 213 \mathrm{~cm} / \mathrm{s}, \mathrm{p}<0.05)$. In both groups, baPWV rose as the number of risk factors for atherosclerosis increased, but baPWV was higher in the OSAS group than in the control group even in a comparison of individuals entirely free from risk factors $(1,400 \pm 200 \mathrm{~cm} / \mathrm{s}$ vs $1,198 \pm 79 \mathrm{~cm} / \mathrm{s}, \mathrm{p}<0.05)$.

Conclusion The condition of OSAS itself is considered a possible risk factor for atherosclerosis. We believe that the usefulness of baPWV as an index of atherosclerosis merits further study in the frequently observed cases of OSAS complicated by cardiovascular disease.

(Internal Medicine 43: 184-188, 2004)
\end{abstract}

Key words: obstructive sleep apnea syndrome, pulse wave velocity, atherosclerosis

\section{Introduction}

Obstructive sleep apnea syndrome (OSAS) is linked with increased hypertension, ischemic heart disease, heart failure, and strokes (1). However, there are few studies concerning atherosclerosis in OSAS patients. Pulse wave velocity (PWV) is known to be an indicator of arterial stiffness $(2,3)$, and has been regarded as a marker reflecting vascular damage $(4,5)$. Although recently a simple non-invasive automatic measurement of brachial-ankle PWV (baPWV) has been described and the validity and reproducibility of this new technique have been reported (6), there have been no studies to assess the significance of baPWV in OSAS patients.

In this study the OSAS group and healthy control group were compared with respect to complications of atherosclerotic risk factors such as hypertension, obesity, hypercholesterolemia, smoking, and impaired glucose tolerance. Also the values of baPWV as an indicator of atherosclerosis were compared in the OSAS group and healthy control group, and the significance of these atherosclerotic risk factors on baPWV was verified in the OSAS group.

\section{Materials and Methods}

\section{Study subjects}

Subjects were 104 OSAS patients (92 men and 12 women, mean age, $53.3 \pm 9.5$ years) examined in our department from June 2002 to July 2003 for complaints such as snoring, difficulty in breathing during sleep, and daytime sleepiness, and

From the Second Department of Internal Medicine Faculty of Medicine, Kagoshima University, Kagoshima and *the Department of Internal Medicine, Kagoshima-kouseiren Hospital, Kagoshima

Received for publication August 25, 2003; Accepted for publication December 1, 2003

Reprint requests should be addressed to Dr. Hiroyuki Nagahama, the Second Department of Internal Medicine Faculty of Medicine, Kagoshima University, 8-35-1 Sakuragaoka, Kagoshima 890-8520 
whose apnea hypopnea index (AHI) was 5 per hour or higher in the polysomnograph (PSG) screening. Their mean AHI was $41.7 \pm 27.1 / \mathrm{h}$. These subjects were studied comparatively with 104 healthy control subjects (92 men and 12 women, mean age, $53.3 \pm 7.7$ years) comprising physical examination recipients matched for age, sex, and body mass index. All healthy control subjects were not on medication and had no medical history of sleep disordered breathing, atherogenic diseases, cardiovascular diseases, renal insufficiency, or other diseases requiring medical treatment. The institutional ethical committee approved the study and all patients gave their informed consent.

\section{Polysomnography}

The PSG apparatus used was the Somnostar $\alpha$ sleep analysis and observation system (SensorMedics Corporation, Yorba Linda, California.) Apnea and hypopnea were assessed by American Academy of Sleep Medicine (AASM) guidelines, with apnea defined as a 10-second or longer continuous cessation of breathing, and hypopnea defined as a $50 \%$ or greater decrease in airflow for 10 seconds or longer, or a less than $50 \%$ decrease in airflow accompanied by an event ranging from arousal response to a $3 \%$ or greater drop in arterial blood oxygen saturation.

\section{baPWV}

baPWV was measured using a volume-plethymographic apparatus (form PWV/ABI; Colin Co., Ltd., Komaki, Japan). This instrument records the phonocardiogram, electrocardiogram, and volume pulse form and arterial blood pressure at both the left and right brachia and ankles. baPWV was calculated by time-phase analysis between the right brachial and volume waveforms at both ankles.

The distance between the right brachium and ankle was estimated based on body height. Figure 1 depicts the pulse wave at the right brachium and ankle in a 70-year-old man

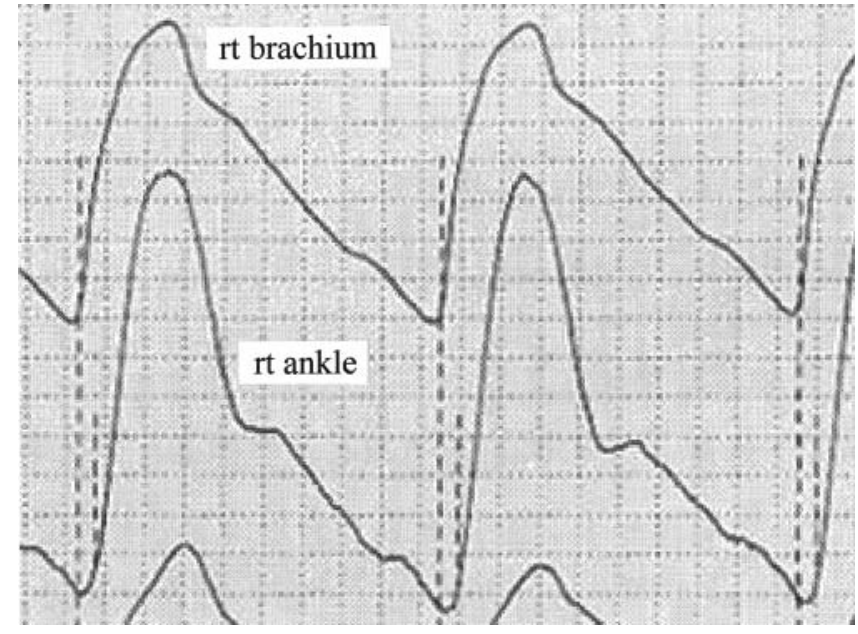

Figure 1. The pulse wave at right brachium and ankle in a 70year-old man with obstructive sleep apnea syndrome who showed the highest baPWV value in the present study $(3,091$ $\mathrm{cm} / \mathrm{s}$ ).

with OSAS who showed the highest bapwv value in this study $(3,091 \mathrm{~cm} / \mathrm{s})$. Since there was a significant positive correlation between left and right baPWV in both the OSAS group ( $\mathrm{r}=0.931, \mathrm{p}<0.0001)$ and the control group $(\mathrm{r}=0.961$, $\mathrm{p}<0.0001$ ), we used a mean value between left and right baPWV during analysis (Fig. 2A, B).

\section{Methods}

Comparison of OSAS group and control group

The OSAS group and control group were compared with respect to complications of hypertension, obesity, hypercholesterolemia, smoking, and impaired glucose tolerance. Comparison was made regarding mean systolic blood

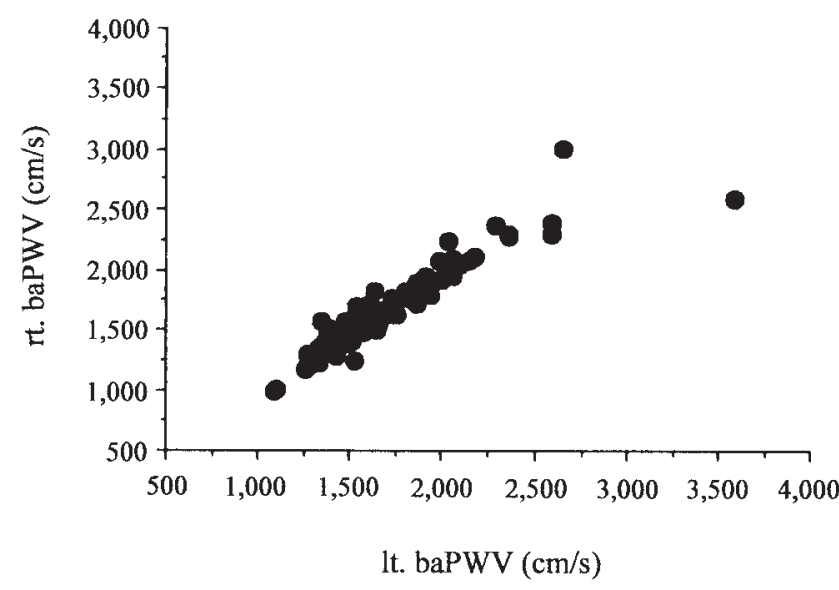

A. OSAS group

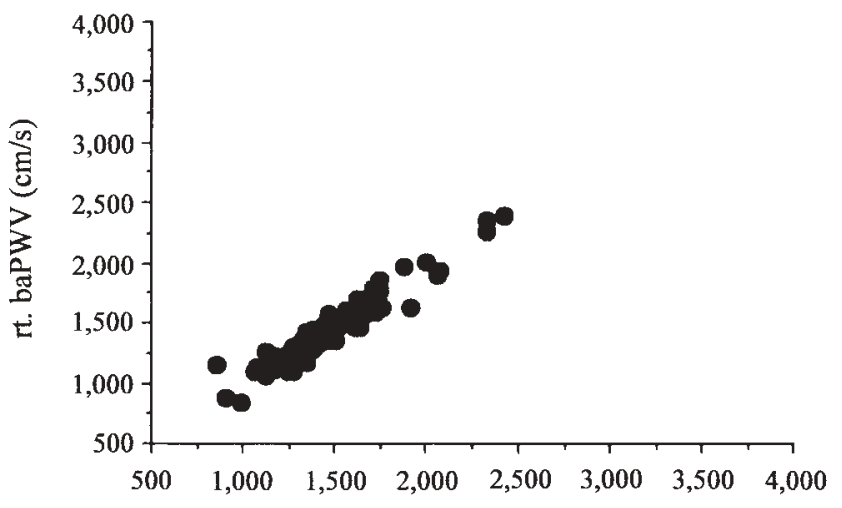

lt. baPWV $(\mathrm{cm} / \mathrm{s})$

Figure 2. Correlation between left and right baPWV in OSAS group and control group. 
NAGAHAMA et al

Table 1. Comparison of Rates of Atherosclerotic Risk Factors between OSAS and Control Group

\begin{tabular}{lccc}
\hline & $\begin{array}{c}\text { OSAS group } \\
\mathrm{n}=104\end{array}$ & $\begin{array}{c}\text { Control group } \\
\mathrm{n}=104\end{array}$ & p-value \\
\hline Hypertension & $53.8 \%$ & $13.5 \%$ & $<0.0001$ \\
Hypercholesterolemia & $34.6 \%$ & $40.4 \%$ & $\mathrm{~ns}$ \\
Obesity & $68.3 \%$ & $70.2 \%$ & $\mathrm{~ns}$ \\
Smoking & $23.1 \%$ & $31.7 \%$ & $\mathrm{~ns}$ \\
Impaired glucose tolerance & $37.5 \%$ & $44.2 \%$ & $\mathrm{~ns}$ \\
\hline
\end{tabular}

OSAS: obstructive sleep apnea syndrome.

pressure, mean diastolic blood pressure, mean body mass index, mean total cholesterol, mean fasting blood glucose, and mean baPWV. Comparisons were also made between 48 OSAS group cases and 90 control group cases free from hypertension, which has a major impact on PWV.

\section{Correlation of baPWV and risk factors for atherosclerosis}

In the OSAS group, correlations were investigated between baPWV and age, BMI, systolic and diastolic blood pressure, total cholesterol, \% time spent with $\mathrm{SpO}_{2}<90 \%$ and AHI.

Comparison of baPWV and degree of risk factors for atherosclerosis

In the OSAS group and the control group, with respect to the number of risk factors for atherosclerosis, including hypertension, hypercholesterolemia, impaired glucose tolerance, smoking, and obesity, the subjects were divided into three groups; no risk group, low risk group with 1-2 risk factors, and high risk group with 3-5 risk factors. Hypertension was defined as systolic blood pressure of $140 \mathrm{mmHg}$ or higher or diastolic blood pressure of $90 \mathrm{mmHg}$ or higher, or use of antihypertensive medication. Hypercholesterolemia was defined as $220 \mathrm{mg} / \mathrm{dl}$ or higher; impaired glucose tolerance was defined as fasting blood glucose of $110 \mathrm{mg} / \mathrm{dl}$ or higher; smoking was defined as current smoking behavior; and obesity was defined as BMI of 25 or higher.

\section{Data analysis}

Data are presented as mean \pm SD unless otherwise stated.

Intergroup comparison was by $\chi^{2}$ independence testing; difference in mean values was tested by the Welch's t-test; and bivariate correlation was by Pearson correlation coefficient with significance at a critical level of $5 \%$ or lower. The medical statistics package Stat View 5.0 was used for statistical analysis.

\section{Results}

\section{Comparison of OSAS group and control group}

In the OSAS group, complication rates for hypertension, obesity, hypercholesterolemia, smoking, and impaired
Table 2. Anthropometric Data of OSAS and Control Groups

\begin{tabular}{lccc}
\hline & $\begin{array}{c}\text { OSAS group } \\
(\mathrm{n}=104)\end{array}$ & $\begin{array}{c}\text { Control group } \\
(\mathrm{n}=104)\end{array}$ & p-value \\
\hline Age $(\mathrm{yr})$ & $53.3 \pm 9.5$ & $53.3 \pm 7.7$ & n.s. \\
BMI $\left(\mathrm{kg} / \mathrm{m}^{2}\right)$ & $26.1 \pm 2.4$ & $26.2 \pm 2.3$ & n.s. \\
TC $(\mathrm{mg} / \mathrm{dl})$ & $208.2 \pm 30.2$ & $216.4 \pm 36.5$ & n.s. \\
FBS $(\mathrm{mg} / \mathrm{dl})$ & $109.2 \pm 22.5$ & $101.3 \pm 13.6$ & $<0.01$ \\
SBP $(\mathrm{mmHg})$ & $135.0 \pm 15.0$ & $125.5 \pm 14.2$ & $<0.0001$ \\
DBP $(\mathrm{mmHg})$ & $83.7 \pm 10.5$ & $77.9 \pm 9.6$ & $<0.0001$ \\
baPWV $(\mathrm{cm} / \mathrm{s})$ & $1,645 \pm 349$ & $1,436 \pm 278$ & $<0.0001$
\end{tabular}

Data are presented mean \pm SD. OSAS: obstructive sleep apnea syndrome, BMI: body mass index, TC: plasma level of total cholesterol, FBS: fasting blood glucose, SBP: systolic blood pressure, DBP: diastolic blood pressure, baPWV: brachial-ankle pulse wave velocity, n.s.: not significant.

glucose tolerance were $53.8 \%, 68.3 \%, 34.6 \%, 23.1 \%$, and $37.5 \%$ respectively. In the control group, the same rates were $13.5 \%, 70.2 \%, 40.4 \%, 31.7 \%$, and $44.2 \%$ (Table 1). Complications of hypertension were significantly higher in the OSAS group. There was no significant difference in obesity, hypercholesterolemia, smoking, and impaired glucose tolerance. Values for systolic blood pressure, diastolic blood pressure, and fasting blood glucose were also significantly higher in the OSAS group (Table 2). The baPWV among the 104 OSAS patients was $1,645 \pm 349 \mathrm{~cm} / \mathrm{s}$, substantially exceeding the adopted reference value of $1,400 \mathrm{~cm} / \mathrm{s}$. The baPWV in the control group was $1,436 \pm 278 \mathrm{~cm} / \mathrm{s}$, and the value in the OSAS group was significantly higher. Cases free from hypertension numbered 48 in the OSAS group and 90 in the control group, and among these individuals baPWV was $1,453 \pm 216 \mathrm{~cm} / \mathrm{s}$ and $1,374 \pm 213 \mathrm{~cm} / \mathrm{s}$, respectively, reflecting a significantly higher value in the OSAS group in this case too (Table 3).

Correlation of baPWV and risk factors for atherosclerosis

Correlation coefficients between baPWV and age, BMI, systolic blood pressure, diastolic blood pressure, total cholesterol, \% time spent with $\mathrm{SpO}_{2}<90 \%$, AHI, and fasting blood glucose were $0.429,-0.049,0.629,0.596,-0.104,0.043$, 
Table 3. Comparison of baPWV Between 48 OSAS Group Cases and 90 Control Group Cases Free from Hypertension

\begin{tabular}{lccc}
\hline & $\begin{array}{c}\text { 48 OSAS } \\
\text { group cases }\end{array}$ & $\begin{array}{c}90 \text { control } \\
\text { group cases }\end{array}$ & p-value \\
\hline Age $(\mathrm{yr})$ & $51.0 \pm 9.4$ & $52.8 \pm 7.7$ & ns. \\
baPWV $(\mathrm{cm} / \mathrm{s})$ & $1,453 \pm 216$ & $1,374 \pm 213$ & $<0.05$ \\
\hline
\end{tabular}

OSAS: obstructive sleep apnea syndrome.

0.09 and 0.142 , respectively, reflecting significant correlations to age, systolic blood pressure, and diastolic blood pressure (Table 4).

Comparison of baPWV and degree of risk factors for atherosclerosis

In the OSAS group, the baPWV of the no risk group, low risk group, and high risk group were $1,400 \pm 200 \mathrm{~cm} / \mathrm{s}$, $1,605 \pm 330 \mathrm{~cm} / \mathrm{s}, 1,751 \pm 369 \mathrm{~cm} / \mathrm{s}$, respectively. In the control group, the baPWV values were $1,198 \pm 79 \mathrm{~cm} / \mathrm{s}, 1,377 \pm$ $207 \mathrm{~cm} / \mathrm{s}, 1,549 \pm 330 \mathrm{~cm} / \mathrm{s}$, respectively. As the degree of risk factors for athelosclerosis increased, baPWV also increased in both the OSAS group and the control group. Even in the same risk group, baPWV was higher in the OSAS group, and even in the no risk group, baPWV in the OSAS group was $1,400 \pm 200 \mathrm{~cm} / \mathrm{s}$, higher than the control group value (Table 5).

\section{Discussion}

In a major epidemiological study of sleep breathing disorders conducted in the U.S., the Sleep Heart Health Study, approximately $16 \%$ of cases suffered from myocardial infarction, angina, heart failure, or strokes (7), but there have been few studies of the cause of these conditions and resulting atherosclerosis because to date there is no simple and precise observational method.

In our study, the baPWV of 104 OSAS patients was distinctly higher than that in the control group, although the potential confounding influences of age, sex, and body mass index on baPWV were minimized by recruiting control sub-
Table 4. Correlation Coefficients between baPWV and Other Clinical Variables in OSAS Group

\begin{tabular}{lrr}
\hline \multicolumn{1}{c}{ Correlates } & \multicolumn{1}{c}{$\mathrm{r}$} & \multicolumn{1}{c}{$\mathrm{p}$} \\
\hline Age & 0.429 & $<0.0001$ \\
BMI & -0.049 & 0.623 \\
Systolic blood pressure & 0.629 & $<0.0001$ \\
Diasystolic blood pressure & 0.596 & $<0.0001$ \\
Total cholesterol & -0.104 & 0.2959 \\
\%Time spent below 90\% $\mathrm{SpO}_{2}$ & 0.043 & 0.6639 \\
Apnea hypopnea index & 0.09 & 0.3641 \\
FBS & 0.142 & 0.1516 \\
\hline
\end{tabular}

OSAS: obstructive sleep apnea syndrome, BMI: body mass index, FBS: fasting blood glucose.

jects who were matched for age, sex, and body mass index. OSAS is prevalent among middle-aged males with a tendency for obesity and it is also regarded as prevalent among patients with hypertension, hypercholesterolemia, diabetes, and obesity, which are deemed risk factors for atherosclerosis. In this study, complications of hypertension were significantly higher in the OSAS group as were values for systolic blood pressure, diastolic blood pressure and fasting blood glucose. Although body mass index was not significantly higher in the OSAS group because of adjustment, the body mass index in the OSAS group was $26.1 \pm 2.4 \mathrm{~kg} / \mathrm{m}^{2}$, substantially exceeding the diagnostic criteria for obesity in Japan $25 \mathrm{~kg} / \mathrm{m}^{2}$. The combination of such risk factors may have resulted in high baPWV. OSAS is closely linked to the cluster of cardiovascular risk factors known as "syndrome X" (8), including systemic hypertension, central obesity, insulin resistance and hyperlipidemia. Wilcox et al proposed a new concept, "syndrome Z", which was "syndrome X" plus OSAS and predicted that aside from co-existent risk factors, untreated OSAS would be associated with an additional independent cardiovascular risk (9). Therefore, here baPWV values were investigated with respect to the number of risk factors.

Comparison of baPWV in the present study with regard to various degrees of risk factors showed that baPWV was significantly higher in the OSAS group than in the control group even when the degree of risk factors was equal and

Table 5. Comparison of baPWV and Number of Risk Factors in OSAS Group

\begin{tabular}{|c|c|c|c|c|c|c|c|c|}
\hline & \multicolumn{3}{|c|}{ OSAS } & \multicolumn{3}{|c|}{ Control } & \multicolumn{2}{|c|}{ p-value } \\
\hline & Age (years) & $\mathrm{baPWV}(\mathrm{cm} / \mathrm{s})$ & $\mathrm{n}$ & Age (years) & baPWV $(\mathrm{cm} / \mathrm{s})$ & $\mathrm{n}$ & Age & baPWV \\
\hline No risk group & $52.9 \pm 5.6$ & $1,400 \pm 200$ & 7 & $45.7 \pm 9.6$ & $1,198 \pm 79$ & 6 & ns & $<0.05$ \\
\hline Low risk group & $52.9 \pm 10.2$ & $1,605 \pm 330$ & 59 & $52.8 \pm 6.7$ & $1,377 \pm 207$ & 56 & ns & $<0.0001$ \\
\hline High risk group & $54.0 \pm 9.0$ & $1,751 \pm 369$ & 38 & $54.9 \pm 8.2$ & $1,549 \pm 330$ & 42 & ns & $<0.05$ \\
\hline
\end{tabular}

OSAS: obstructive sleep apnea syndrome, No risk group: subjects free from risk factors of atherosclerosis, Low risk group: subjects with 1or 2 risk factors of atherosclerosis, High risk group: subjects with 3 and more risk factors of atherosclerosis. 
even when there was no risk factor. Additionally, baPWV was significantly higher in the OSAS group than in the control group even in a comparison of individuals free from hypertension, which has a major impact on baPWV.

Based on these results, we predicted that the condition of OSAS itself would be a risk factor for atherosclerosis among OSAS patients, one which would further advance atherosclerosis when combined with other risk factors. Plasma vascular endothelial growth factor (VEGF) is a potent angiogenic cytokine, and recent studies have shown that VEGF may also contribute to the atherogenic process itself. VEGF induces monocyte activation and migration (10), modulates the growth of smooth muscle cells (11), and is closely related to the progression of coronary atherosclerosis in humans (12, 13) and the extent of carotid stenosis (14).

Schulz et al have reported that repeated nighttime hypoxia elevates plasma VEGF in OSAS patients, and they hypothesize that the alterations of the VEGF system in severe OSAS may have an impact on the development of cardiovascular abnormalities in these patients (15). In the present study, we did not observe a correlation between $\mathrm{AHI}$ or $\mathrm{SpO}_{2}<90 \%$ time versus baPWV, but we also believe that OSAS itself can constitute a risk factor for atherosclerosis as a result of repeated nighttime hypoxia, and we believe that further study is needed.

Much interest has been shown over the past few decades in PWV as a noninvasive method for assessing atherosclerotic diseases. Blacher et al (16) reported that the value of PWV in subjects with atherosclerotic disease was significantly higher than that in normal subjects. They also reported that the higher the PWV value, the higher the risk of cardiovascular disease in normal subjects.

Although PWV has been reported to be a useful method for assessing atherosclerosis, the past methods used for measurement of carotid-femoral PWV have required complex techniques and have not demonstrated reproducibility. Form ABI/PWV, a recently developed device, enables simultaneous measurement of blood pressure levels of limbs and can calculate ABI and baPWV in a short time. Moreover, reproducible results can be obtained regardless of the operators technique (intraobserver reproducibility is $10 \%$ ) (6). Therefore, this test might predict the risk of atherosclerosis and it's high values may mean that the atherosclerotic process is already well established. Kwok et al (17) reported that 30 children with primary snoring who did not show significant apnea or hypopnea had significantly higher blood pressure and PWV than healthy control subjects matched for age, sex, and body mass index and predicted that long-term cardiovascular health might be jeoparadized by chronic elevation of systolic blood pressure and reduction of arterial distensibility. To the best of our knowledge, the present study is the first to assess the significance of baPWV on OSAS patients. We believe that periodic monitoring and follow-up of baPWV as a comprehensive index of atherosclerosis is necessary for the prevention of complications among OSAS patients, among whom cardiovascular disease is a frequent complication.

\section{References}

1) Leung RS, Bradley TD. Sleep apnea and cardiovascular disease. Am J Respir Crit Care Med 164: 2147-2165, 2001.

2) Lehmann ED. Clinical value of aortic pulse-wave velocity measurement. Lancet 354: 528-529, 1999.

3) Asmar R, Benetos A, Topouchian J, et al. Assessment of arterial distensibility by automatic pulse wave velocity measurement: validation and clinical application studies. Hypertension 26: 485-490, 1995.

4) Cohn JN. Vascular wall function as a risk marker for cardiovascular disease. J Hypertens 17 (Suppl 5): S41-S44, 1999.

5) van Popele NM, Grobbee DE, Bots ML, et al. Association between arterial stiffness and atherosclerosis: the Rotterdam Study. Stroke 32: 454-460, 2001.

6) Yamashina A, Tomiyama H, Takeda K, et al. Validity, reproducibity, and clinical significance of noninvasive brachial-ankle pulse wave velocity measurement. Hypertens Res 25: 359-364, 2002.

7) Shahar E, Whitney CW, Redline S, et al. Sleep-disordered breathing and cardiovascular disease: cross-sectional results of the Sleep Heart Health Study. Am J Respir Crit Care Med 163: 19-25, 2001.

8) Reaven GM. Banting lecture 1988 Role of insulin resistance in human disease. Diabetes 37: 1595-1607, 1988.

9) Wilcox I, McNamara SG, Collins FL, Grunstein RR, Sullivan CE. "Syndrome Z": the interaction of sleep apnoea, vascular risk factors and heart disease. Thorax 53: S25-S28, 1998.

10) Clauss $M$, Gerlach $M$, Gerlach $H$, et al. Vascular permeability factor: a tumor-derived polypeptide that induces endothelial cell and monocyte procoagulant activity, and promotes monocyte migration. J Exp Med 172: $1535-1545,1990$.

11) Doi K, Itoh H, Komatsu $Y$, et al. Vascular endothelial growth factor suppresses C-type natriuretic peptide secretion. Hypertension 27 (3 pt. 2): 811-815, 1996.

12) Inoue $M$, Itoh $H$, Ueda $M$, et al. Vascular endothelial growth factor (VEGF) expression in human coronary atherosclerotic lesions. Possible pathophysiological significance of VEGF in progression of atherosclerosis. Circulation 98: 2108-2116, 1998.

13) Clauss M, Weich $H$, Breier G, et al. The vascular endothelial growth factor receptor Flt-1 mediates biological activities. J Biol Chem 271: 17629-17634, 1996.

14) Lee SH, Bae HR, Jeong SJ, et al. Circulating levels of interleukin-8 and vascular endothelial growth factor in patients with carotid stenosis. J Korean Med Sci 16: 198-203, 2001.

15) Schulz R, Hummel C, Heinemann S, Seeger W, Grimminger F. Serum levels of vascular endothelial growth factor are elevated in patients with obstructive sleep apnea and severe nighttime hypoxia. Am J Respir Crit Care Med 165: 67-70, 2002.

16) Blacher J, Asmar R, Djane S, London GM, Safar ME. Aortic pulse wave velocity as a marker of cardiovascular risk in hypertensive patients. Hypertension 33: 1111-1117, 1999.

17) Kwok KL, Ng DK, Cheung YF. BP and arterial distensibility in children with primary snoring. Chest 123: 1561-1566, 2003. 